\title{
AUTOMATED HIGH RESOLUTION MEASUREMENT OF HELIOSTAT SLOPE ERRORS
}

\author{
Steffen Ulmer ${ }^{1}$, Tobias März ${ }^{1}$, Christoph Prahl', Wolfgang Reinalter ${ }^{1}$, Boris Belhomme ${ }^{2}$ \\ 1 German Aerospace Center (DLR), Institute of Technical Thermodynamics, Solar Research, Plataforma Solar de Almería \\ (PSA), 04200 Tabernas, Spain, Phone: +34 950 265320, Fax: +34 950 260315, e-mail: steffen.ulmer@dlr.de \\ ${ }^{2}$ German Aerospace Center (DLR), Institute of Technical Thermodynamics, Solar Research, 51170 Cologne, Germany
}

\begin{abstract}
A new optical measurement method that simplifies and optimizes the mounting and canting of heliostats and helps to assure their optical quality before commissioning of the solar field was developed. This method is based on the reflection of regular patterns in the mirror surface and their distortions due to mirror surface errors. The measurement has a resolution of about one million points per heliostat with a measurement uncertainty of less than $0.2 \mathrm{mrad}$ and a measurement time of about one minute per heliostat. The system is completely automated and allows the automatic measurement of an entire heliostat field during one night. It was extensively tested at the CESA-1 heliostat field at the Plataforma Solar de Almería. Comparisons of flux simulations based on the measurement results with real flux density measurements were performed. They showed an excellent agreement and demonstrated in a striking manner the high measurement accuracy and high grade of detail in the simulation achieved by this technique.
\end{abstract}

Keywords: heliostat, optical quality, optical measurement, deflectometry, ray tracing, flux measurement.

\section{Introduction}

The heliostat field is the major cost component of a solar thermal tower power plant and the optical quality of the heliostats has a significant impact on the field efficiency and thus on the performance of the power plant. It is therefore a permanent goal to decrease their manufacturing cost but at the same time maintain or even improve their optical quality. To accomplish that, economical and accurate measurement systems are needed to detect optical deficiencies in facets and support structures, to allow precise and optimized canting without sun and to determine the overall optical quality of single heliostats and entire fields for acceptance tests.

Since the early eighties canting and quality assessment of heliostats was mainly done by observation of the focal spot produced by the heliostat on a flat white surface close to the receiver area. Specialized measurement systems with digital cameras and specific evaluation algorithms were developed to quantify and automate this task [1,2]. Although this method delivers a graphic and reliable result it has two main drawbacks: it depends on clear-sky conditions and it only allows to determine an overall quality parameter (beam quality), but it is not possible to resolve and assign local surface errors.

For facet canting without sun a total station can be used. All four corners of each facet are measured with a prism and from these points in space the facet normal and its necessary correction is calculated. This method is used in recent years at the Plataforma Solar de Almería and in several collector assembly lines. However, this method requires considerable manual work and considers the information of only four points per facet. Any local errors at these points are translated to the orientation of the entire facet. Manufacturing errors of the facets themselves cannot be measured. The same applies to the canting method with a rotating laser proposed by Monterreal [3].

For the measure of local shape deviation of solar concentrators photogrammetry can be used [4]. However, as the measured surface has to be equipped with a large number of target points which are used as individual surface measurement points, the measurement is a time consuming task which is not appropriate for the measurement of large numbers of mirrors. A further drawback of this method is that the slopes of the reflective surface are calculated from coordinates of points in space. High-resolution measurements imply 
short distances between the measured points and tiny but inevitable measurement deviations can lead to significant errors in the local slope calculated. To overcome this dilemma several methods that directly measure surface slopes instead of surface coordinates were developed:

CIEMAT proposed to use the reflection of a star in the mirror at night and move the heliostat to get enough information to calculate the local surface normals at various points on the reflector [5]. Again, this method depends on clear sky conditions, although at night, and its use is a time consuming task, especially when high spatial resolution is desired.

The so-called V-shot measurement system developed by Sandia and NREL measures the local slopes of a mirror by scanning it with a laser beam, detecting the point of incidence of the reflected beam and calculating the resulting surface normal [6]. So far this system was only applied for dishes and parabolic troughs where it is sufficiently accurate. However, it might be difficult to adapt it to heliostat measurements due to the large distances and the necessary extremely high pointing precision of the laser that comes along with it. Also, scanning at high resolution is time consuming.

A measurement principle called deflectometry or fringe reflection has been coming up in the last decade in various applications where specular surfaces need to be qualified such as windscreens, glossy automobile parts, corrective lenses for glasses and so on $[7,8]$. This method uses known regular stripe patterns whose reflection in the mirror is observed by a camera. The deformations of the stripe pattern in the reflection are then used to evaluate the local slopes of the mirror. This method was first introduced in the qualification of concentrating solar collectors by the German Aerospace Center (DLR) $[9,10]$ and is more recently also used by other groups, although in the qualification of other concentrators than heliostats [11,12].

Based on this basic principle, DLR has developed a new measurement system for the special case of heliostats. The declared aim was to develop a system that allows measuring surface slopes with high resolution and with high accuracy, and is suitable for large surfaces, rapid and easy to set-up.

\section{Measurement}

\section{Measurement system}

From daily observations it is known that smallest irregularities in a mirror surface cause the reflected image to distort considerably. Hence, an observer that studies the reflection of a regular pattern can detect irregularities in the slope of a specular surface that would often be imperceptible on a dull surface. This principle can be used to accurately measure local slopes for mirror surfaces.

The chosen set-up for measuring heliostats in a solar tower power plant is shown in Figure 1. A projector situated in the heliostat field projects the stripe patterns on a white target surface at the tower at night. A digital camera located on top of the tower takes images of the heliostats which are oriented the way that the stripe patterns can be seen in the reflection. If the camera position, the heliostat position and the target position are known and if it is achieved to clearly identify each point $M(i, j)$ of the distorted pattern seen in the heliostat and assign its corresponding position $D(i, j)$ on the target, the local normal vector can be calculated from the law of reflection:

$$
n=\frac{r-i}{|r-i|}
$$

With the normalized vectors $i$ for the incident ray, $r$ for the reflected ray and $n$ for the surface normal. 


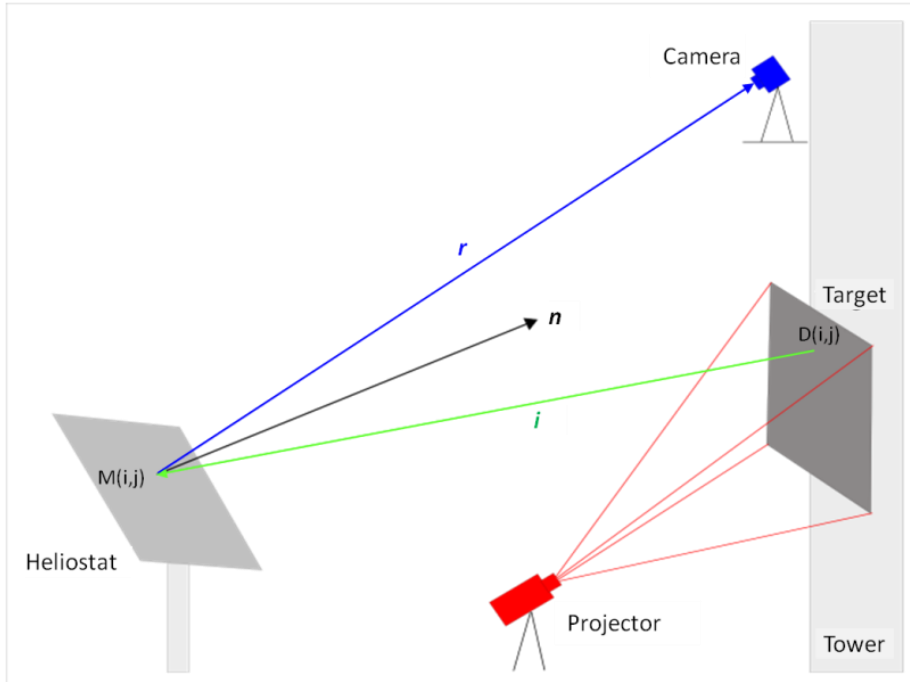

Figure 1: Measurement set-up used for heliostat measurements in the field

The unambiguous identification of the reflected target points is achieved by codifying the target surface with a series of stripe patterns. Vertical stripes are used to codify the target in $\mathrm{x}$-direction and horizontal stripes are used to code it in y-direction. Stripe patterns with sinusoidal brightness variations are used to codify the position in each direction. Figure 2 shows an example picture of a projected horizontal stripe pattern on the target surface (left) and the corresponding pattern as it is seen from the camera in the reflection of the heliostat.
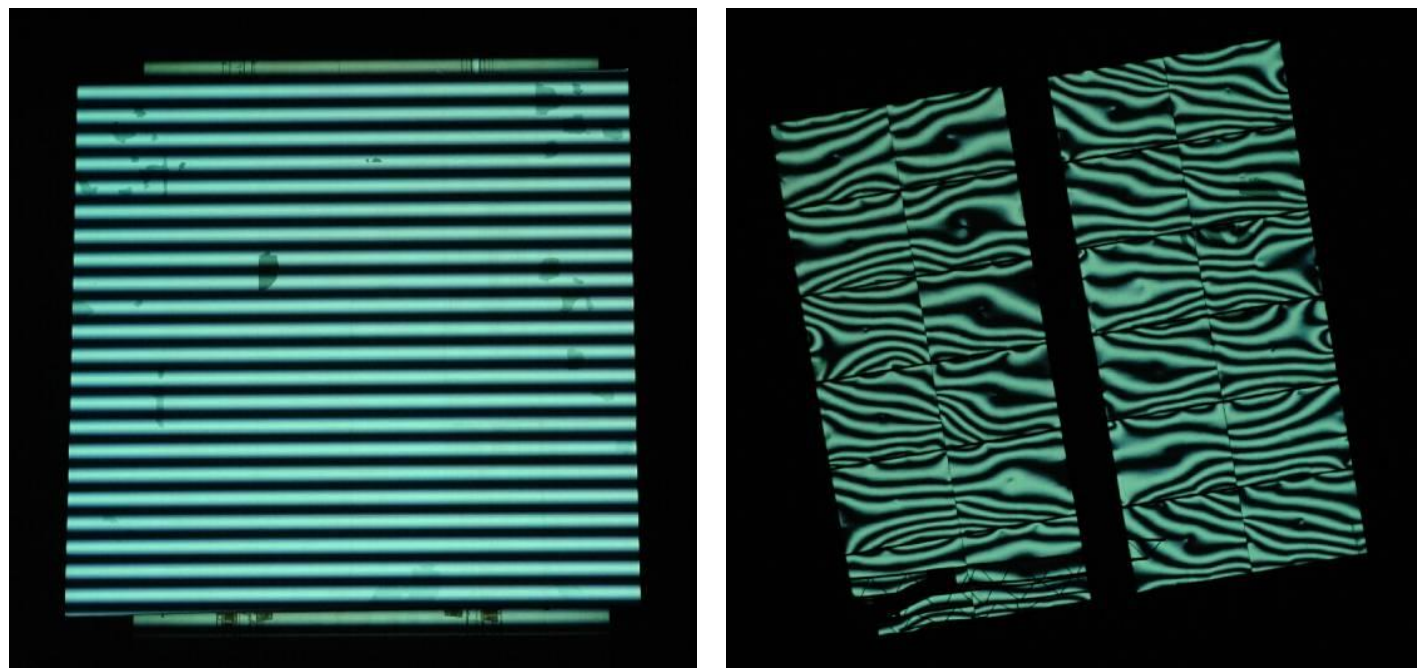

Figure 2: Example of a regular horizontal stripe pattern reflected in a heliostat

From the brightness (grey level) seen at a certain point, the corresponding phase of the sinus wave can be assigned. However, this is ambiguous for phase angles larger than $\pm \pi / 2$ as the same grey values appear repeatedly. Within the period of 0 to $2 \pi$, this can be resolved by using four patterns with a phase shift of $\pi / 2$ between each.

$$
\operatorname{phase}(i, j)=\operatorname{atan}\left(\frac{G V_{4}(i, j)-G V_{2}(i, j)}{G V_{1}(i, j)-G V_{3}(i, j)}\right)
$$

This also increases the accuracy of the determined phase and makes it independent of local brightness alterations. The ambiguity between multiples of $2 \pi$ can be solved by using additional patterns with longer wavelengths (Figure 3). The phase information of the longer wavelength is used to identify the phase interval of the shorter wavelength pattern. The accuracy in the determination of the phases in the long wavelength series must be higher than the phase interval of the shorter wavelength series to be identified. Additional 
stripe pattern series with shorter wavelengths can be used to increase final precision. This method of codification is called hierarchical phase shift (HPS).

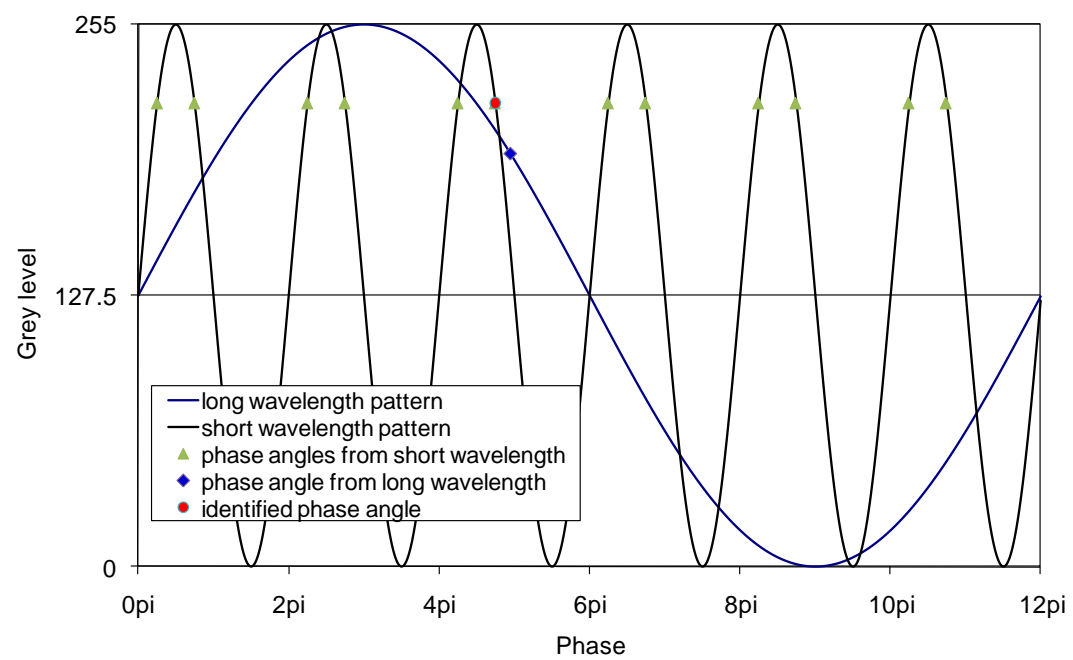

Figure 3: Decoding of target position coded with sinusoidal stripe patterns with different wavelengths

A computer algorithm developed in Matlab® evaluates the images, calculates the surface normal vectors and visualizes the results. It takes into account and corrects all known systematic error influences such as distorted projection of stripe patterns due to oblique projection angle and projector lens distortions, nonlinearity of projector and camera, background lighting, inhomogeneous reflection properties of mirror and target, perspective rectification, lens aberrations, etc.

Results

Figure 4 shows an example measurement result of a heliostat at the Plataforma Solar de Almería (PSA). The result is given as deviation of local slope angle from ideal slope angle in milliradians (mrad) in x-direction (azimuth) in the left graph and in y-direction (elevation) in the right graph. The deviations are in the range of $\pm 5 \mathrm{mrad}$. Positive deviations in $\mathrm{x} / \mathrm{y}$-direction correspond to tilt of the normal vector towards the negative $\mathrm{x} / \mathrm{y}$ direction. Certain systematic deviations can be observed: in the facets generally the $\mathrm{x}$-deviation is positive in the left half and negative in the right half. This corresponds to a longer focal length in $\mathrm{x}$ than the ideal one. The same behavior can be observed for the entire heliostat. In y-direction, especially within the right half, there are some facets with positive or negative deviations in the entire facet area which corresponds to deviations in the facet alignment. This misalignment could be corrected by re-canting the facets. There are also some local deviations visible (most visibly in the facet in the upper left corner), probably due to stresses from mirror mounting and bending. 

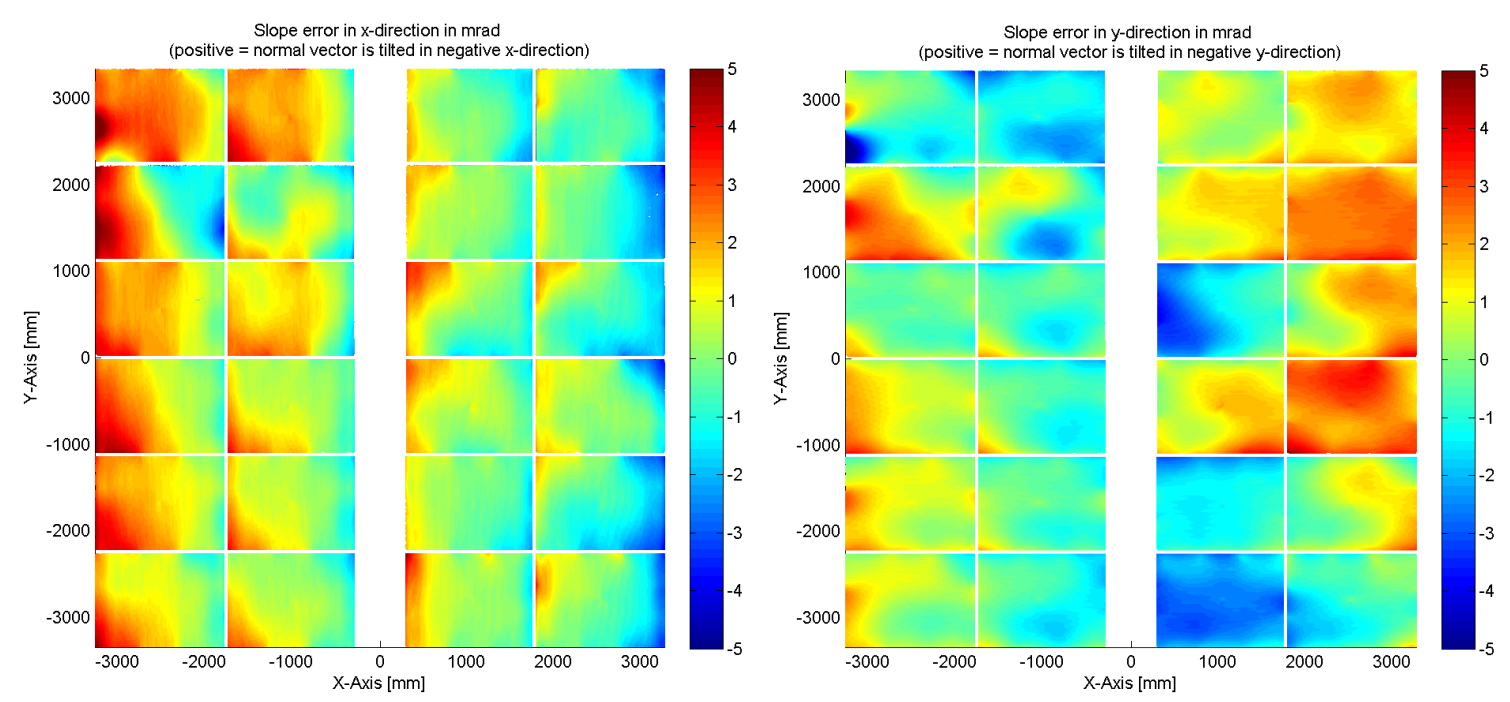

Figure 4: Measurement result, displayed as slope deviations from ideal surface in azimuth (left) and elevation (right) in milliradians

The high resolution data are further processed for interpretation. The standard deviation of the slope deviation within an individual facet corresponds to the deformation or shape deviation of the facet itself. The mean deviation of the slope deviation within an individual facet corresponds to the deviation in facet alignment. The measured focal lengths of the facets are determined by a parabolic fit to the data within one facet. Table 1 shows an example of these values for the measurement shown above. These values can be used for adjustments of facet canting and for control of facet focal lengths. Heliostat facets are often made from flat glass that is slightly bent by a center screw that draws the facet back. For facets that allow adjustment of the focal length by such a center screw, the corresponding center displacement for adjustment can be calculated.

\begin{tabular}{|c|c|c|c|c|c|c|c|c|c|c|c|c|c|c|}
\hline \multicolumn{5}{|c|}{ Tilt in $\mathrm{x}$-direction [mrad]: } & \multicolumn{5}{|c|}{ Tilt in y-direction [mrad]: } & \multicolumn{5}{|c|}{ Measured focal length [m]: } \\
\hline Row/Col & 1 & 2 & 3 & 4 & Row/Col & 1 & 2 & 3 & 4 & Row/Col & 1 & 2 & 3 & 4 \\
\hline 1 & 1.96 & 1.32 & -0.46 & -1.11 & 1 & -1.25 & -1.72 & 0.32 & 1.25 & 1 & 116.8 & 141.5 & 145.0 & 126.7 \\
\hline 2 & 0.46 & -0.18 & -0.16 & -1.27 & 2 & 1.33 & -0.58 & 1.00 & 2.08 & 2 & 242.0 & 97.8 & 131.5 & 155.8 \\
\hline 3 & 1.32 & 0.68 & -0.17 & -1.15 & 3 & -0.29 & -0.68 & -1.88 & 1.13 & 3 & 152.2 & 135.3 & 138.6 & 137.7 \\
\hline 4 & 1.13 & 0.15 & 0.06 & -0.54 & 4 & 1.02 & -0.57 & 1.18 & 2.43 & 4 & 189.0 & 135.1 & 142.0 & 134.8 \\
\hline 5 & 1.23 & 0.01 & -0.56 & -1.55 & 5 & 0.87 & -0.40 & -1.34 & 0.38 & 5 & 158.4 & 143.9 & 145.5 & 146.4 \\
\hline 6 & 0.57 & -0.12 & -0.04 & -1.00 & 6 & 0.45 & -0.83 & -2.45 & -1.06 & 6 & 143.2 & 129.0 & 133.3 & 143.6 \\
\hline
\end{tabular}

Table 1: Measurement results of individual facets derived from high resolution data

The same values can be determined for the entire heliostat. The shown heliostat has a standard slope deviation of $1.35 \mathrm{mrad}$ in $\mathrm{x}$-direction and $1.44 \mathrm{mrad}$ in y-direction, which corresponds to a total RMS-value of $1.98 \mathrm{mrad}$. The measured focal length of the heliostat is $116.2 \mathrm{~m}$, design focal length is $104.1 \mathrm{~m}$.

\section{Uncertainty analysis}

The deflectometric measurement principle has the advantage that the reflexion information is used as direct input of the measurement which leads to high accuracies in the measured surface slopes at high spatial resolution. Due to the numerous transformations and corrections during the evaluation procedure the uncertainty analysis of the measurement method is a complex task. To cope with this a mathematical model that considers all known uncertainties and that completely reproduces the evaluation process was developed. It allows the prediction of the expected error for a certain measurement set-up, the calculation of the local error of performed measurements and is a valuable tool for the system improvement as error budgets can be analyzed in detail. At present, a resolution of about 1000x1000 measurement points per heliostat with a local measurement error of below $0.2 \mathrm{mrad}$ is reached. 


\section{Automation}

The measurement procedure and evaluation was completely automated. An industrial type CCD camera from Lumenera ${ }^{\circledR}$ together with a computer controlled pan/tilt head and motor zoom lens allow completely automatic scans of entire heliostat fields. The projected stripe patterns are remotely controlled and the camera shutter triggered accordingly. The different components of the measurement system communicate over a wireless network, so no cabling except electric power is necessary (Figure 5). The measurement time for one heliostat is about one minute, so several hundred heliostats can be measured during one night.
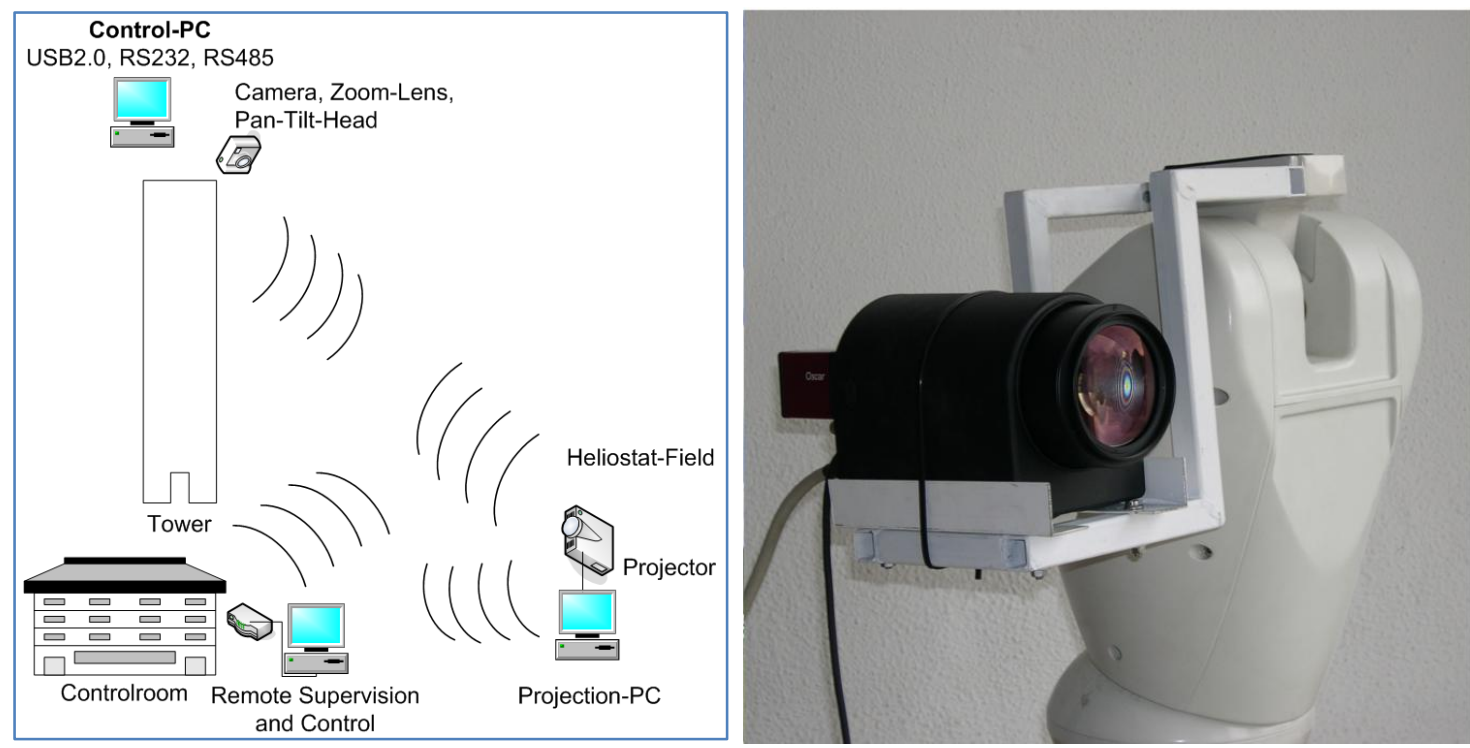

Figure 5: Sketch of the automatic measurement system with wireless communication (left) and camera system with motor zoom on pan/tilt head with removed housing (right).

The evaluation process is independent from the image acquisition process. A configuration file with all necessary evaluation parameters has to be created by the user. Some start values for automatic edge detection and thresholds have to be determined which is usually done during the evaluation of the first heliostat. The rest of the images can be processed in batch mode without further user interaction. The evaluation time for each heliostat depends on the selected resolution and the options chosen, but is usually in the range of about one minute. That means that the measurements taken during one night can be evaluated in one day. Compared to existing measurement methods described in the introduction this method offers significant gain in speed and handling.

One limitation of the described measurement method is that the facets need to be sufficiently pre-aligned to guarantee that the reflection of the stripe patterns on the target can be seen in the entire heliostat. This is not case if errors exceed a certain limit. Yet, as described in [13] these cases can be covered by a different optical measurement method that uses the same hardware and set-up, although with less accuracy.

\section{Ray Tracing Simulation and Validation}

In recent years DLR also developed a new ray tracing program named STRAL (Solar Tower Raytracing Laboratory [14]) that efficiently processes large numbers of high resolution data sets obtained from above described heliostat measurements. It allows fast and accurate simulations of the flux distributions delivered by the heliostats and can be used for assessment of the measurement results and for optimization of heliostat field operation using dynamic aimpoint strategies.

Measurements of flux distributions of real heliostats at PSA were used to validate the deflectometry measurement accuracy and the ray tracing model by comparison. In order to take into account as many influences as possible, a group of four heliostats partly blocked and shaded by the heliostats in front was chosen (Figure 6). 


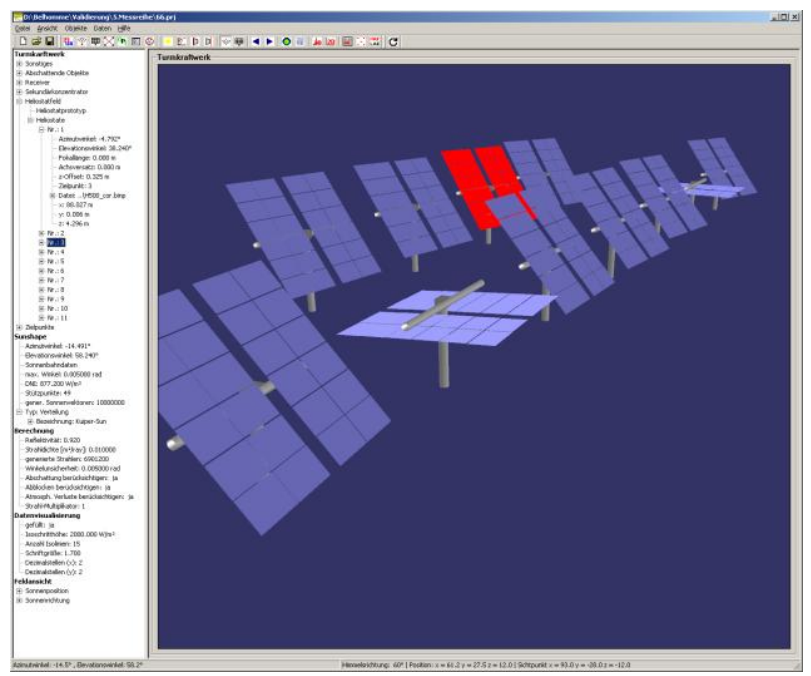

Figure 6: Screenshot of ray tracing program STRAL with heliostat set-up used for validation

A flux density measurement with the four heliostats in the back row pointing to four different points on the flat measurement target at the tower and with the heliostats of the front row in the shown positions was performed. Also, deflectometry measurements of all four heliostats were done and the corresponding data files for STRAL generated. Figure 7 shows the measured flux distribution of the four heliostats (bold lines) and the ray tracing result (thin lines) in an overlay. The agreement between both distributions is very good which means that local surface deviations, as well as shading and blocking are correctly reflected in measurement and simulation.

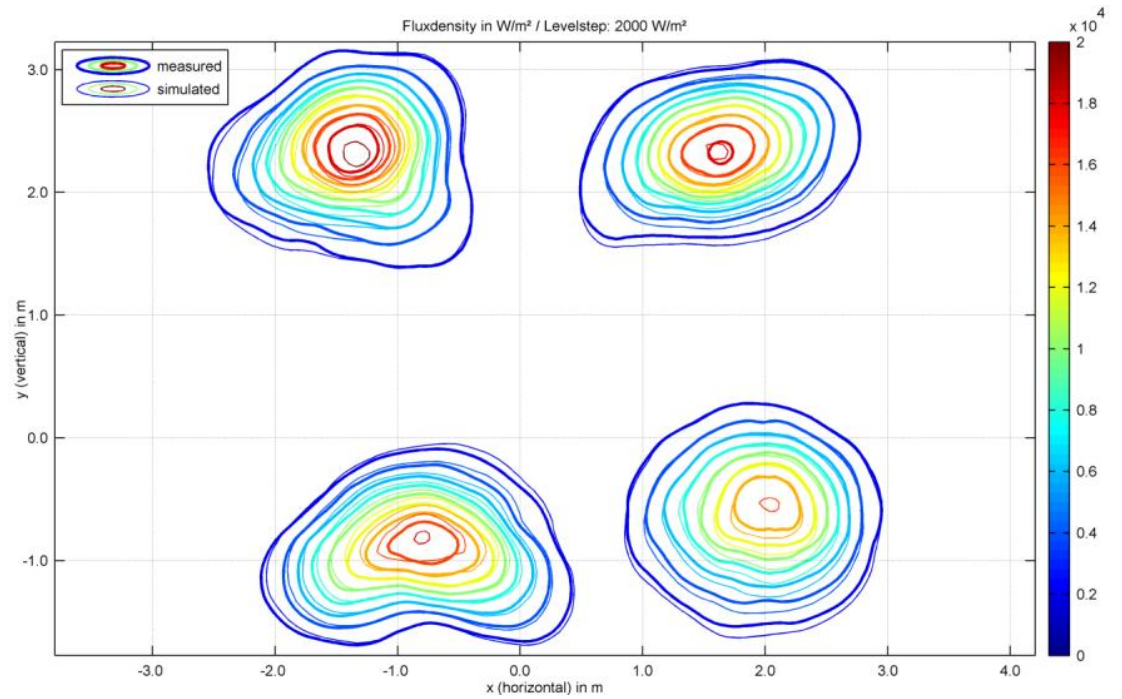

Figure 7: Measured flux distribution of the four heliostats (bold lines), and simulated flux distribution of four heliostats based on measured deflectometry data (thin lines).

This type of highly accurate ray tracing simulations based on high resolution measurement data allow much more realistic predictions and optimizations of the heliostat field performance than in earlier simulation models where shape deviations of heliostats were approximated with a single value for a Gaussian slope error distribution [15].

\section{Summary and Outlook}

The presented optical measurement system allows economic and automatic measurements of concentrator slope deviations of entire heliostat fields with high resolution and accuracy. This can be used to check and optimize concentrator quality, canting of facets and the optical quality of entire heliostat fields. Combined 
with the ray tracing code STRAL it is possible to accurately predict the heliostat field performance and improve heliostat field operation using dynamic aimpoint strategies. The described measurement method can also be used to measure other solar concentrators such as dishes, troughs and individual facets.

\section{Acknowledgements}

Financial support from the German ministry for the environment, nature conservation and nuclear safety within the contract 03UM0068 is gratefully acknowledged.

\section{References}

[1] R. Monterreal: Optical Comparison ASM 150 and GM 100A, in "Proceedings of the 10th Task III Meeting within the IEA SolarPACES on "Solar Technology and Applications, SolarPACES Technical Report III - 6/97", Sandia, Albuquerque, 15.9.1997

[2] A. Imenes, W. Stein, J. Hinkley, R. Benito, R. Bolling, P. Schramek, S. Ulmer: Ray Tracing and Flux Mapping as a Design and Research Tool at the National Solar Energy Centre, Paper number S06-81, ANZSES 2006 conference (Australia and New Zealand Solar Energy Society), September 13th-15th, 2006, Canberra, Australia.

[3] R. Monterreal: A New On-Axis Canting Method for Faceted Heliostats, Solar Thermal Concentrating Technologies, proceedings of the 8th International Symposium, October 6-11, 1996, Köln, Germany, Deutsche Forschungsanstalt für Luft- und Raumfahrt e.V. Manfred Becker/Manfred Böhmer (Hrsg.), Müller, Heidelberg, 1997

[4] K. Pottler, E. Lüpfert, G. Johnston, M. Shortis, Photogrammetry: A Powerful Tool for Geometric Analysis of Solar Concentrators and Their Components, Journal of Solar Energy Engineering, February 2005, Volume 127, Issue 1, pp. 94-101.

[5] F. Arqueros, A. Jimenez, A. Valverde: A novel procedure for the optical characterization of solar concentrators, Solar Energy 75 (2003) 135-142.

[6] S.A. Jones, D.R. Neal, J.K. Gruetzner, R.M. Houser, R.M. Edgar, J. Kent, T.J. Wendelin: VSHOT: A Tool for Characterizing Large, Imprecise Reflectors, International Symposium on Optical Science Engineering and Instrumentation, 1996, Denver, CO.

[7] S. Kammel: Deflectometry for quality control of specular surfaces. Technisches Messen tm 70, pp. 1938, April 2003

[8] M.C. Knauer, J. Kaminski, G. Häusler: Phase Measuring Deflectometry: a new approach to measure specular free-form surfaces. Optical Metrology in Production Engineering, Proc. SPIE 5457, 2004

[9] S. Ulmer, P. Heller, W. Reinalter: Slope Measurements of Parabolic Dish Concentrators Using Color Codified Targets. Proceedings of the 13th SolarPACES International Symposium on Concentrated Solar Power and Chemical Energy Technologies, June 20-23, 2006, Seville, Spain, ISBN: 84-7834-519-1.

[10] S. Ulmer, M. Röger: Automatisierte hochaufgelöste Vermessung der Spiegelfehler von Heliostaten. 10. Kölner Sonnenkolloquium „F*E mit hochkonzentrierter Strahlung“, 21. Juni 2007, DLR, Köln-Porz.

[11] A. Heimsath, W. Platzer, T. Bothe, L. Wansong: Characterization of Optical Components for Linear Fresnel Collectors by Fringe Reflection Method, Proceedings of Solar Paces Conference, 2008, Las Vegas, NV.

[12] C. Andraka, S. Sadlon, B. Myer, K. Trapeznikov, C. Liebner: Rapid Reflective Facet Characterization Using Fringe Reflection Techniques. Proceedings of the Energy Sustainability 2009, July 19-23, 2009, San Francisco, California, USA.

[13] M. Röger, C. Prahl, S. Ulmer: Fast Determination of Heliostat Shape and Orientation by Edge Detection and Photogrammetry, Proceedings of the 14th Biennial CSP SolarPACES Symposium, March 4-7, 2008, Las Vegas, NV.

[14] B. Belhomme, R. Pitz-Paal, P. Schwarzbözl, S. Ulmer: A new fast ray tracing tool for high-precision simulation of heliostat fields, Journal of Solar Energy Engineering, August 2009, Vol. 131, Issue 3.

[15] P. Garcia, A. Ferriere, J.-J. Bezian: Codes for Solar Flux Calculation Dedicated to Central Receiver System Application: A Comparative Review, Solar Energy, 2007, 82(3), pp. 189-197. 